Historic, Archive Document

Do not assume content reflects current scientific knowledge, policies, or practices. 



\section{FALL OF 1910}

\section{Trade List of Nursery Stock.}

OFFERED FOR SALE ATUIA

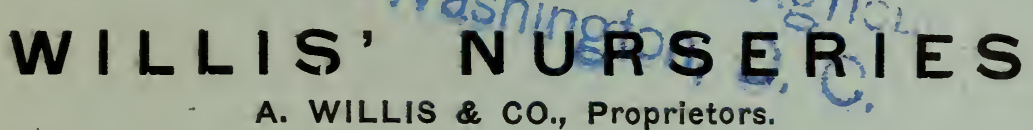

A. WILlis \& CO., Proprietors.

OTTAWA, KANSAS.

The following is all thrifty and well grown No. 1 stock of the sizes and ages named. Packing will be done at cost, but no charge will be made for drayage to the depot or express office. For stock sold terms are cash or satisfactory security before shipment.

\section{ENTOMOLOGIST'S CERTIFICATE WITH EVERY SHIPMENT.}

\section{APPLE.}

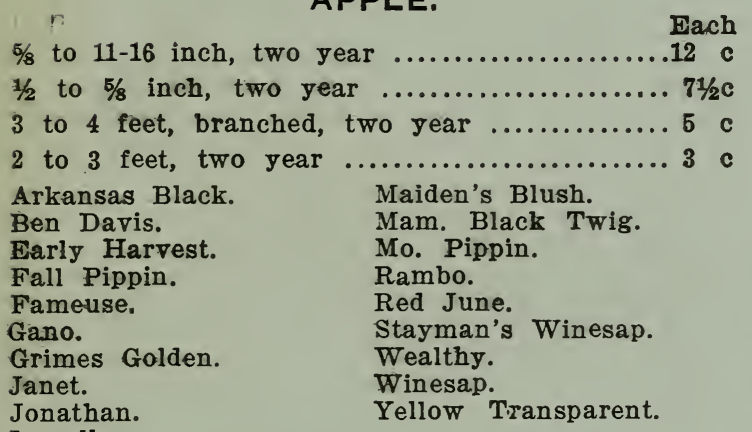

\section{Apple-One year.}

4 to 6 feet

Each

3 to 4 feet

Arkansas Black.

Ben Davis.

Gano.

Grimes Golden.

Huntsman.

Jonathan.

Maiden Blush.

Mam. Black Twig.

\section{CHERRIES.}

$3 / 4$ inch and up, two year

$5 / 8$ to $3 / 4$ inch, two year

$1 / 2$ to $5 / 8$ inch, two year

Mo. Pippin.

Rome Beauty.

Stayman's Winesap.

W. W. Pearmain.

Winesap.

Wolf River.

Yellow Bellflower.

York Imperial.

$\% / 8$ to $1 / 2$ inch, two year

Black Tartarian.

Dyehouse.

Early Richmond.

Wragg.

\section{STANDARD PEARS.}

$3 / 4$ inch and up, two year

Each

$5 / 8$ to $3 / 4$ inch, two year ......................11c

$1 / 2$ to $5 / 8$ inch, two year $\ldots \ldots \ldots \ldots \ldots \ldots \ldots \ldots .6 \mathrm{c}$

Bartlett.

Clapp's Favorite.

Flemish Beauty.

Howell.

Garber.
DWARF PEARS.

11-16 inch and up, two year Each

$5 / 8$ to $11-16$ inch, two year ................. $71 / 2 \mathrm{c}$

$1 / 2$ to $5 / 8$ inch, two year ...................

Bartlett.

Clapp's Favorite.

Duchess.

\section{PLUM ON PLUM.}

Each

..............14c

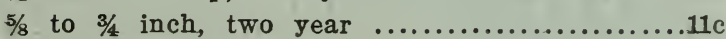

$1 / 2$ to $5 / 8$ inch, two year ......................

Abundance. Burbank.

Bradshaw. Lombard.

German Prune. Shipper's Pride.

PEACH

Each

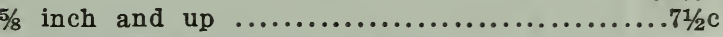

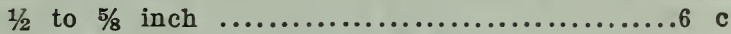

$3 / 8$ to $1 / 2$ inch ............................. $11 / 2 \mathrm{c}$

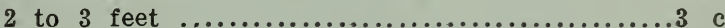

Alexander. Foster.

Amsden. Greensboro.

Ark. Traveler. Hales Early.

Blood Cling. Heath Cling.

Bokara. Lemon Cling.

Carman. Old Mixon Cling.

Champion. Old Mixon Free.

Crawford's Early. Salway.

Crawford's Late. Sneed.

Crosby.

Elberta.

Fitzgerald.

Stump World.

Triumph.

Wonderful.

\section{APRICOTS.}

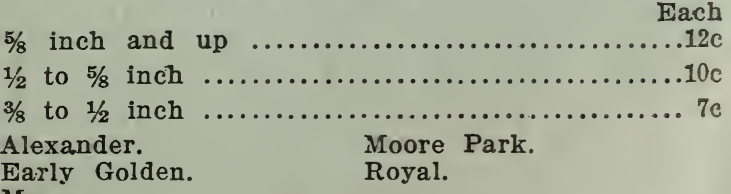

Early Golden.

Mason.

QUINCES.

$5 / 8$ inch and up, two year ..................18c

$1 / 2$ to $5 / 8$ inch, two year $\ldots \ldots \ldots \ldots \ldots \ldots \ldots \ldots \ldots \ldots \ldots$

Champion. Orange. 
Per M.

Brighton, 1 year No, $1 \ldots \ldots \ldots \ldots \ldots \ldots \ldots . . . .100$

Concord, 1 year No. 1 ................... 15.00

Moore's Early, 1 year No. 1 ................ 30.00

Niagara, 1 year No. 1 .......................... 25.00

Pocklington, 1 year No. 1 ............... 20.00

Worden, 1 year No. 1 ................. 30.00

\section{GOOSEBERRIES. : : Each}

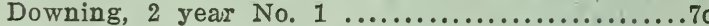

Houghton, 2 year No. 1 ..........................6c

Industry, 2 year No. 1 ...........................

\section{CURRANTS}

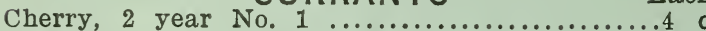

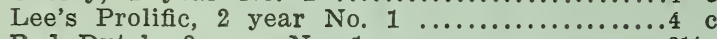

Red Dutch, 2 year No. $1 \ldots \ldots \ldots \ldots \ldots \ldots \ldots \ldots 3^{1 / 2} \mathrm{c}$

White Grape, 2 year No. 1 ...................4c

Cardinal ................................. Per $\$ 10.00$

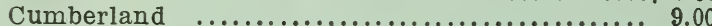

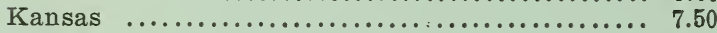

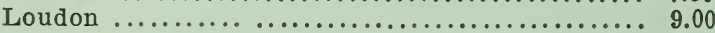

BLACKBERRIES. Per M. Early Harvest...$\ldots \ldots \ldots \ldots \ldots \ldots \ldots \ldots \ldots \ldots \ldots 10.00$ Rathbun .................................. 15.00

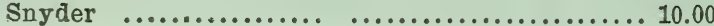

STRAWBERRIES. Per $M$
Leading Varieties

Bederwood.

Bubach.

Capt. Jack.

Charles Downing.

Crescent.

Excelsior.

Gandy.

Glendale.

$$
\begin{aligned}
& \text { Glen Mary. } \\
& \text { Greenville. } \\
& \text { Jesse. } \\
& \text { Sharpless. } \\
& \text { Senator Dunlap. } \\
& \text { Sample. } \\
& \text { Warfield. }
\end{aligned}
$$

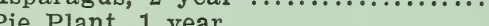

作

Horse Radish ...................\$1.00 per 100

\section{ORNAMENTAL TREES}

Each

Ash, 6 to 8 feet ............................

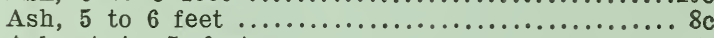

Ash, 4 to 5 feet .............................

Catalpa, 10 to 12 feet ..............................

Catalpa, 8 to 10 feet ..............................

Catalpa, 5 to 6 feet $\ldots \ldots \ldots \ldots \ldots \ldots \ldots \ldots \ldots \ldots \ldots \ldots$ c

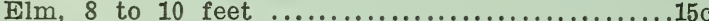

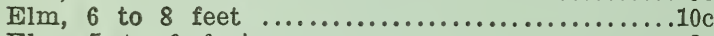

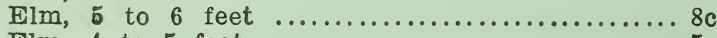

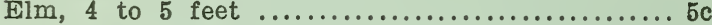

Elm, 3 to 4 feet $\ldots \ldots \ldots \ldots \ldots \ldots \ldots \ldots \ldots \ldots \ldots \ldots \ldots \ldots \ldots$

Locust, Black, 10 to 12 feet $\ldots \ldots \ldots \ldots \ldots \ldots \ldots \ldots \ldots . .15 \mathrm{c}$

Locust Black, 8 to 10 fsst ...................

Locust, Black, 6 to 8 feet ..................10 c

Locust, Black, 5 to 6 feet ..................... 7c

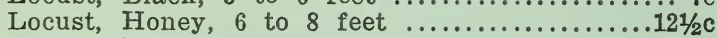

Locust, Honey, 5 to 6 feet $\ldots \ldots \ldots \ldots \ldots \ldots \ldots \ldots$. $10 \mathrm{c}$

Locust, Honey, 4 to 5 feet ................. $8 \mathrm{c}$

Maple, Soft, 8 to 10 feet ...........................

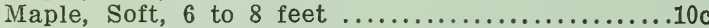

Maple, Soft, 5 to 6 feet $\ldots \ldots \ldots \ldots \ldots \ldots \ldots \ldots$.

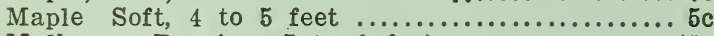

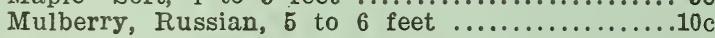

Mulbersy, Russian, 4 to 5 feet $\ldots \ldots \ldots \ldots \ldots \ldots, 7 \mathrm{c}$

Mulberry, Russian, 3 to 4 feet .............. 5c

Poplar Carolina, $11 / 4$ to $1 \frac{1}{2}$ inch caliper .........15c

Poplar Carolina, 10 to 12 feet ................10 c

Poplar Carolina, 8 to 10 feet ................ 8c

Poplar Carolina 6 to 8 feet ...................6. $6 \mathrm{c}$

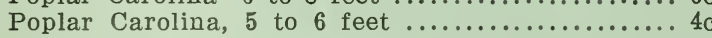

Poplar Norway, 8 to 10 feet $\ldots \ldots \ldots \ldots \ldots \ldots \ldots \ldots \ldots \ldots \ldots \ldots$

Poplar Norway, 6 to 8 feet $\ldots \ldots \ldots \ldots \ldots \ldots \ldots \ldots \ldots \ldots$.

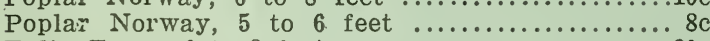

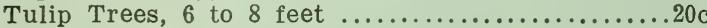

Tulip Trees, 5 to 6 feet ....................

\section{VINES.}

Each

Clematis, Jackmanii, 2 year No. 1 ...........20 c

Clematis, Henryii, 2 year No. 1 ................20

Clematis, Mad Ed Andre, 2 year No. 1 ........20c

Clematis, Paniculata, 2 year No. $1 \ldots \ldots \ldots \ldots . .10 \mathrm{c}$

Honeysuckle, Hall's Japan ......................

Trumpet Vine .................................. 8c

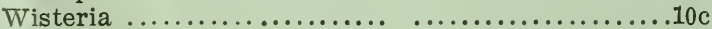

SHRUBS.

Althea Pink 2 to 3 feet

年,

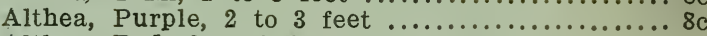
Althea, Red, 2 to 3 feet .................... 8c Althea, White, 2 to 3 feet $\ldots \ldots \ldots \ldots \ldots \ldots \ldots \ldots . .6$ Deutzia, 2 to 3 feet .......................10 Hydrangea, P. G., 2 to 3 feet .................... Hydrangea, P. G., 18 to 24 inches ............. 8c Honeysuckle, Upright, 2 to 3 feet ................ Japan Quince, 2 to 3 feet .......................... $8 c$ Japan Quince, 3 to 4 feet ......................10

Lilac Purple, 2 to 3 feet $\ldots \ldots \ldots \ldots \ldots \ldots \ldots \ldots, c$

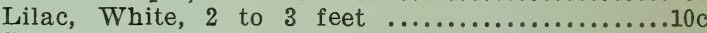

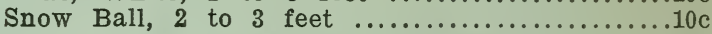
Spirea Anthony Waterer, 12 to 18 inches........10c Spirea Billardi, 2 to 3 feet $\ldots$

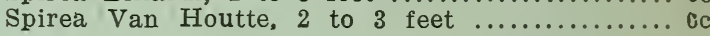

\section{PAEONIAS.}

Each

Pink, Double

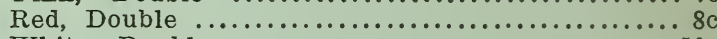

White, Double ..............................

Single, Mixed, fine assortment $\ldots \ldots \ldots \ldots \ldots \ldots$. c

\section{ROSES.}

Climbing Roses No. 1 ..................... $8 \mathrm{c}$ Hybrid Perpetual No. 1 .....................12c

Moss No. 1 ................................... Ramblers No. 1 .....................................

\section{EVERGREENS}

Arbor Vitae, American, 3 feet .................... Arbor Vitae, American, 2 to 3 feet ................10 Arbor Vitae, American, 18 to 24 inches ......... 8c Arbor Vitae, Pyrmidalis, 3 feet .................. Juniper, Irish, 3 feet .........................

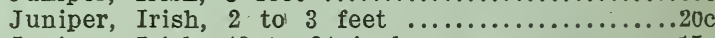

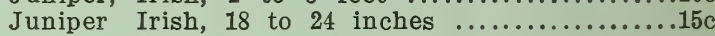
Pine, Austrian, 2 to 3 feet ...................12c Pine, Austrian, 18 to 24 inches ...............10 c Pine, Scotch, 3 feet ............................ Pine, Scotch, 2 to 3 feet ......................10

Pine, Scotch, 18 to 24 inches ................. $8 \mathrm{c}$

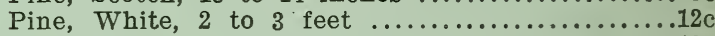

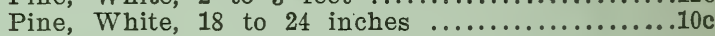

\section{HEDGE.}

Osage Orange, 1 year No. $1 \ldots \ldots \ldots \ldots \ldots \ldots \ldots$. Per 1.25 Osage Orange, 1 year No. $2 \ldots \ldots \ldots \ldots \ldots \ldots \ldots \ldots \ldots . . .1 .00$

Privet California, 18 to 24 inches ...........20.00 Privet California, 12 to 18 inches $\ldots \ldots \ldots \ldots \ldots . . .15 .00$ Privet California, 8 to 12 inches ............... 10.00

\section{FOREST SEEDLINGS}

Per M.

Catalpa Speciosa, 2 to 3 feet $\ldots \ldots \ldots \ldots \ldots \ldots \ldots . . \$ 40$ Catalpa Speciosa, 18 to 24 inches .............. 3.00 Catalpa Speciosa 12 to 18 inches ................ 2.50 Catalpa Speciosa, 6 to 12 inches ............ 1.50

Elm, 18 to 24 inches ....................... 3.50

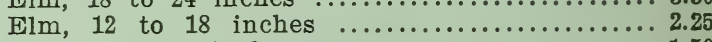

Elm, 6 to 12 inches ...................... 1.50

Locust, Black, 18 to 24 inches .................. 3.00

Locust, Black, 12 to 18 inches ................2.50

Locust, Black, 6 to 12 inches .................... 1.50

Locust, Honey, 12 to 18 inches ..............2.25

Locust, Honey, 6 to 12 inches .............. 1.50

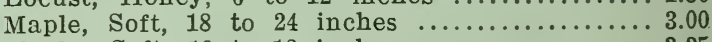

Maple Soft, 12 to 18 inches $\ldots \ldots \ldots \ldots \ldots \ldots \ldots .2 .25$

Maple, Soft, 6 to 12 inches ................ 1.50

\section{SUPPLIES.}

Burlap, $7 \frac{1}{2}$ oz., 40 inches wide .........6c per yd. Labels, iron wired $\ldots \ldots \ldots \ldots \ldots \ldots \ldots . . .75 \mathrm{c}$ per $M$. Rope, Pure Sisal .................... per $1 \mathrm{~b}$.

\section{SCALECIDE.}

Scalecide..................... \$1.25 per gallon.

Printed information and instructions sent on application. 